\title{
A modified quantum adiabatic evolution for the Deutsch-Jozsa problem
}

\author{
Zhaohui Wei and Mingsheng Ying \\ State Key Laboratory of Intelligent Technology and Systems, \\ Department of Computer Science and Technology, Tsinghua University, Beijing, China, 100084
}

\begin{abstract}
Deutsch-Jozsa algorithm has been implemented via a quantum adiabatic evolution by S. Das et al. [Phys. Rev. A 65, 062310 (2002)]. This adiabatic algorithm gives rise to a quadratic speed up over classical algorithms. We show that a modified version of the adiabatic evolution in that paper can improve the performance to constant time.
\end{abstract}

PACS numbers: 03.67.Lx

Quantum computation has attracted a great deal of attention in the recent years, because some quantum algorithms show that the principles of quantum mechanics can be used to greatly enhance the efficiency of computation. Quantum algorithms that have been invented include Deutsch-Jozsa algorithm 1, 2], Shor's algorithm 3] and Grover's algorithm [4]. The first one is what we will discuss in this paper.

Assume that we have a boolean function of the form

$$
f:\{0,1\}^{n} \rightarrow\{0,1\},
$$

and it has been known that the function is either constant (i.e., all outputs are identical) or balanced (i.e., has an equal number of 0's and 1's as outputs). Our task is to decide whether it is constant or not. To solve this problem, any deterministic classical algorithm needs $2^{n} / 2+1$ evaluation of the function $f$, while Deutsch-Jozsa algorithm 1, 2] shows that a quantum computer can solve the same problem with one evaluation.

When these algorithms mentioned above were invented, they were implemented using quantum circuits involving a sequence of unitary operators. We say these algorithms stay within the standard paradigm of quantum computation. Recently, another novel paradigm based on quantum adiabatic evolution has been proposed for quantum computation [5]. In a quantum adiabatic algorithm, the state of the quantum register evolves under a hamiltonian that varies continuously and slowly. At the beginning, we let the state of the system in the ground state of the initial hamiltonian. If the hamiltonian of the system evolves slowly enough, the quantum adiabatic theorem guarantees that the final state of the system will differ from the ground state of the final hamiltonian by a negligible amount. If we encode the solution of the algorithm in the ground state of the final hamiltonian, after the quantum adiabatic evolution we can get the solution with high probability by measuring the final state.

Some quantum algorithms have been reproduced by adiabatic evolutions. In 6] S. Das et al. solved the Deutsch-Jozsa problem by an adiabatic evolution. This adiabatic evolution gives rise to a quadratic speed up over classical algorithms. Recently, the experimental implementation of this adiabatic algorithm has been reported by A. Mitra et al [8]. However, compared to the standard quantum algorithm for this problem, this adiabatic algorithm is not so good and needs to be improved. In this paper, we propose a modified adiabatic evolution for the Deutsch-Jozsa problem based on the one in [6], and we will show that the running time of the modified adiabatic evolution is constant. (We have noted that in 7] M. S. Sarandy and D. A. Lidar have obtained the same result via a different adiabatic evolution.)

For convenience of the readers, we present an overview of adiabatic algorithms. Suppose $H_{0}$ and $H_{T}$ are the initial and the final Hamiltonians of the system. Suppose $|\alpha\rangle$, the ground state of $H_{0}$, is the initial state of the system and $|\beta\rangle$, the ground state of $H_{T}$, is the final state that encodes the solution. Then we let the system vary under the following time dependent Hamiltonian:

$$
H(t)=(1-s) H_{0}+s H_{T},
$$

where $s=s(t)$ is a monotonic function with $s(0)=0$ and $s(T)=1$ ( $T$ is the running time of the evolution). Let $\left|E_{0}, t\right\rangle$ and $\left|E_{1}, t\right\rangle$ be the ground state and the first excited state of the Hamiltonian at time $\mathrm{t}$, and let $E_{0}(t)$ and $E_{1}(t)$ be the corresponding eigenvalues. The adiabatic theorem [9] shows that we have

$$
\left|\left\langle E_{0}, T \mid \psi(T)\right\rangle\right|^{2} \geq 1-\varepsilon^{2},
$$

provided that

$$
\frac{D_{\max }}{g_{\min }^{2}} \leq \varepsilon, \quad 0<\varepsilon \ll 1,
$$

where $g_{\min }$ is the minimum gap between $E_{0}(t)$ and $E_{1}(t)$

$$
g_{\min }=\min _{0 \leq t \leq T}\left[E_{1}(t)-E_{0}(t)\right]
$$

and $D_{\max }$ is a measurement of the evolving rate of the Hamiltonian

$$
D_{\max }=\max _{0 \leq t \leq T}\left|\left\langle\frac{d H}{d t}\right\rangle_{1,0}\right|=\max _{0 \leq t \leq T}\left|\left\langle E_{1}, t\left|\frac{d H}{d t}\right| E_{0}, t\right\rangle\right| .
$$

In the adiabatic evolution of [6], the initial and the final Hamiltonians are

$$
H_{0}=I-|\alpha\rangle\langle\alpha|,
$$




$$
H_{T}=I-|\beta\rangle\langle\beta|,
$$

where

$$
\begin{aligned}
& |\alpha\rangle=\frac{1}{\sqrt{N}} \sum_{i=0}^{N-1}|i\rangle, \quad N=2^{n}, \\
& |\beta\rangle=\mu|0\rangle+\frac{\nu}{\sqrt{N-1}} \sum_{i=1}^{N-1}|k\rangle,
\end{aligned}
$$

with

$$
\begin{gathered}
\mu=\frac{1}{N}\left|\sum_{x \in\{0,1\}^{n}}(-1)^{f(x)}\right|, \\
\nu=1-\mu .
\end{gathered}
$$

To solve the Deutsch-Jozsa problem, after the adiabatic evolution ends we measure the final state of the system. If the measurement yields $|0\rangle, f(x)$ is constant and if the measurement doesn't yield $|0\rangle, f(x)$ is balanced.

In [6], S. Das et al. showed that the running time of the local adiabatic evolution [10] above is

$$
T=O(\sqrt{N})
$$

So, the performance of the adiabatic algorithm for this problem is related to $n$. However, using standard quantum computational techniques to solve the Deutsch-Jozsa problem [1, 2], the quantum computer needs only one evaluation of the function $f$ no matter how big $n$ is. We may guess that the adiabatic evolution can be improved. In fact, we can do this by modifying the adiabatic algorithm as follows.

In the modified adiabatic evolution, we choose the new final state

$$
|\beta\rangle=\frac{\mu}{\sqrt{N / 2}} \sum_{k=0}^{N / 2-1}|2 k\rangle+\frac{\nu}{\sqrt{N / 2}} \sum_{i=0}^{N / 2-1}|2 i+1\rangle,
$$

and $H_{0}, H_{T},|\alpha\rangle, \nu, \mu, H(s)$ don't change. Furthermore, we assume that $s(t)$ is linear in $\mathrm{t}, s=t / T$. Obviously, if the final state of the system is $|\beta\rangle$, we can decide whether the function $f(x)$ is balanced or constant by measuring the system after the adiabatic evolution. If the measurement yields $|i\rangle$ and i is even, $f(x)$ is constant. If the measurement yields $|i\rangle$ and i is odd, $f(x)$ is balanced. Now we prove that the running time of the modified adiabatic evolution is constant.

Firstly, it's easy to prove that

$$
|\langle\alpha \mid \beta\rangle|=\frac{1}{\sqrt{2}} .
$$

According to the claim 7 of [1] (see also 12]), we know that

$$
g_{\min }=\frac{1}{\sqrt{2}}
$$

On the other hand,

$$
D_{\max }=\max _{0 \leq t \leq T}\left|\left\langle\frac{d H}{d t}\right\rangle_{1,0}\right| \leq \frac{1}{T} \max _{0 \leq t \leq T}\left\|\frac{d H}{d s}\right\| .
$$

Substituting $\left\|\frac{d H}{d s}\right\|=\left\|H_{T}-H_{0}\right\| \leq 2$ into Eq.(16), we get

$$
D_{\max } \leq \frac{2}{T}
$$

Substituting Eqs. (15), and (17) in Eq. (3), we have

$$
T \geq \frac{4}{\varepsilon} .
$$

That is to say, the running time of the modified adiabatic evolution is independent of $N$.

In conclusion, we have shown that a modified version of the adiabatic evolution of [6] can solve the Deutsch-Jozsa problem in constant time. This is consistent with the result of standard quantum algorithm based on quantum gates.

We would like to thank Ji Zhengfeng, M. S. Sarandy, and D. A. Lidar for useful discussions.
[1] R. Cleve, A. Ekert, C. Macchiavello, and M. Mosca, Proc. R. Soc. London, Ser. A 454, 339 (1998).

[2] D. Deutsch and R. Jozsa, Proc. R. Soc. London, Ser. A 439, 553 (1992).

[3] Shor. P. W, Proc. 35th Annual Symposium on the Foundations of Computer Science, Shafi Goldwasser, ed., (IEEE Computer Society Press), 121-134 (1994).

[4] L. K. Grover, Phys. Rev. Lett 79, 325 (1997).

[5] E. Farhi, J. Goldstone, S. Gutmann, and M. Sipser, eprint quant-ph/0001106

[6] S. Das, R. Kobes, and G. Kunstatter, Phys. Rev. A 65, 062310(2002).
[7] M. S. Sarandy and D. A. Lidar, e-print quant-ph/0502014

[8] A. Mitra, A. Ghosh, R. Das, A. Patel, and A. Kumar, e-print quant-ph/0503060.

[9] L. I. Schiff, Quantum Mechanics (McGraw-Hill, Singapore, 1955).

[10] J. Roland and N. J. Cerf, Phys. Rev. A 65, 042308 (2002).

[11] D. Aharonov and A. Ta-Shma, e-print quant-ph/0301023

[12] Zhaohui Wei and Mingsheng Ying, e-print quant-ph/0504113 